\title{
Advanced Optical Technologies for Space Exploration (Invited)
}

\author{
Natalie Clark \\ NASA Langley Research Center, Hampton VA, 23681
}

\begin{abstract}
NASA Langley Research Center is involved in the development of photonic devices and systems for space exploration missions. Photonic technologies of particular interest are those that can be utilized for in-space communication, remote sensing, guidance navigation and control, lunar descent and landing, and rendezvous and docking. NASA Langley has recently established a class-100 clean-room which serves as a Photonics Fabrication Facility for development of prototype optoelectronic devices for aerospace applications. In this paper we discuss our design, fabrication, and testing of novel active pixels, deformable mirrors, and liquid crystal spatial light modulators. Successful implementation of these intelligent optical devices and systems in space, requires careful consideration of temperature and space radiation effects in inorganic and electronic materials. Applications including high bandwidth inertial reference units, lightweight, high precision star trackers for guidance, navigation, and control, deformable mirrors, wavefront sensing, and beam steering technologies are discussed. In addition, experimental results are presented which characterize their performance in space exploration systems.
\end{abstract}

Keywords: Star Tracker, Active Optics, Adaptive Optics, Active Pixel Sensors, Liquid Crystals, Wavefront Sensors, Deformable Mirror Device.

\section{Introduction}

Current state-of-the-art commercial star sensors typically weigh 15 pounds, attain 5 to 10 arc second accuracy, and use roughly 10 watts of power. Unfortunately, the current state-of-the-art star sensors do not meet many of NASA's "next generation" spacecraft and instrument needs. Nor do they satisfy the DoD mission needs for light weight, low power star sensors for applications such as micro/nano satellite systems. In this section we summarize our Intelligent Star Tracker, called IntelliStar which offers advantages in speed, size, weight and accuracy. Our IntelliStar system illustrated in figure 1 incorporates adaptive optics in a folded catadioptric telescope in a single, compact robust silicon carbide housing. The deformable mirrors and corrector plate are used to compensate for jitter, misalignment, and aberrations to ensure optical performance. ${ }^{1-4}$

Leveraging our adaptive optics research, our active pixel position sensors have wide dynamic range which enables simultaneous imaging of faint and bright stars in a single image frame. The deformable mirror enables extremely accurate tracking. When coupled with our star matching scheme based on algebraic coding theory, the active optic technologies enable fast accurate star pattern recognition to support guidance, navigation and control (GN\&C). ${ }^{3}$

\subsection{IntelliStar: Intelligent Star Tracker}

A prototype of the folded catadioptric telescope was fabricated and is shown in figure 2. The spherical mirror with a stop at the center of curvature is free of coma, astigmatism and distortion. Due to the symmetry of the system the field is curved. Hence a simple field flattener is used at the plane exiting the optical head to the APPS array. The corrector plate is thin and hence the off-axis aberrations introduced by this correcting plate are negligible due to its small power and location of the stop.

Since the deformable mirror devices are not complete yet we placed an optically flat mirror in place that will be replaced once the deformable mirrors are completed. The prototype was fabricated out of aluminum which has a density near that of silicon carbide as discussed in section 1.4. The corrector optic is a very thin machined corrector plate. This will be replaced by a liquid crystal or polymer wavefront corrector plate in the next generation IntelliStar systems discussed in section 1.2. The prototype optical head shown in figure 2(a) weighs 
less than 1.8 pounds and we anticipate the Next Generation Silicon Carbide IntelliStar to weigh less than 8 ounces. Most of the weight reduction is due to eliminating the primary mirror in our first prototype, a metalized thick lens. Instead, as discussed in section 1.5, the silicon carbide mirror will have an aspect ratio of 1:20 instead of the current 1:5 aspect ratio.

The Prototype was placed on a tripod controlled by two high precision stepper motors as shown in figure 2(b). One motor controls for tip/tilt and one controls roll/yaw rotation. The precision of the stepper motor coarse tracking is from 360 degrees to 0.001 degree. We use this as a course adjustment emulating the position of the spacecraft or to hold the star pattern in the center field of view. The deformable mirrors enable the fine tracking with accuracy of 0.0001 degree to .01 degrees. The unit is currently controlled by a laptop computer but the software/firmware is portable and can be used in virtually any embedded system. We are in the process of developing plug and play modules for the next generation IntelliStar system. ${ }^{1-4}$

Star

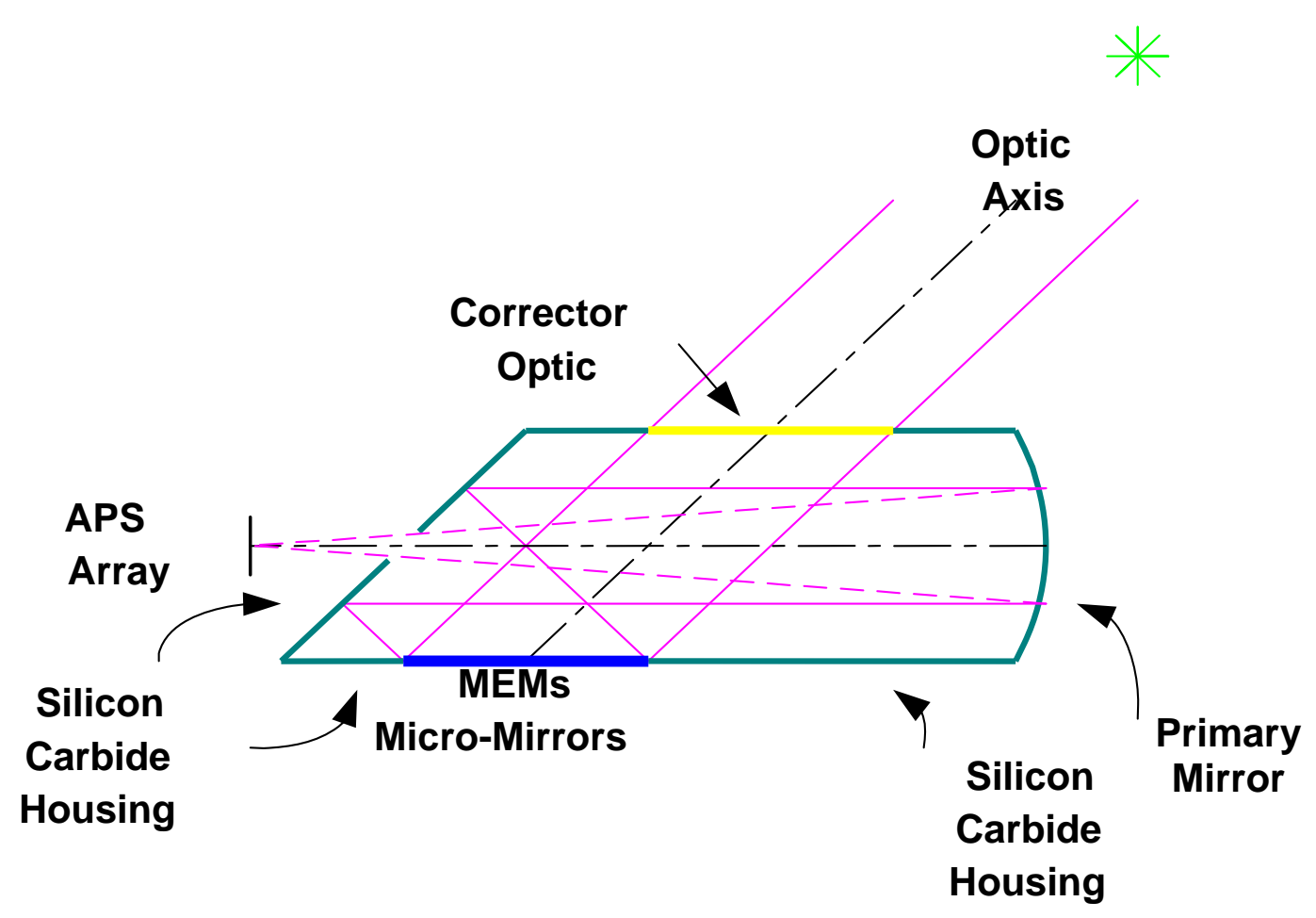

Figure 1. IntelliStar system. Optical Schematic of the Intelligent Star Tracker system. 


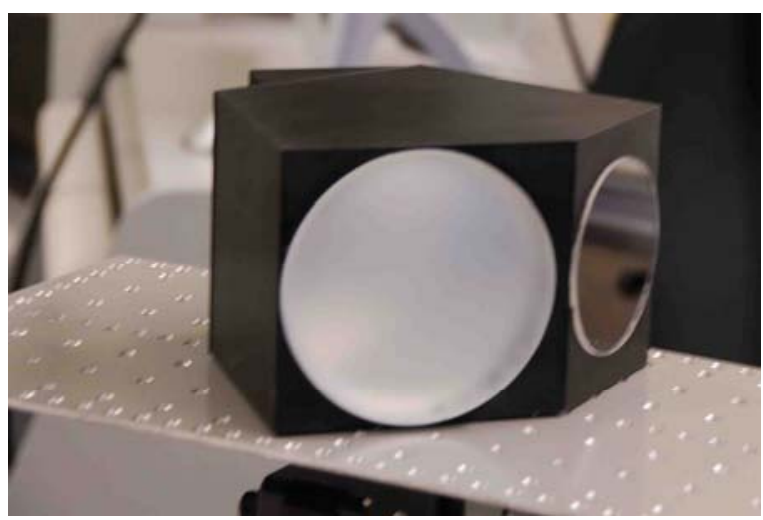

(a)

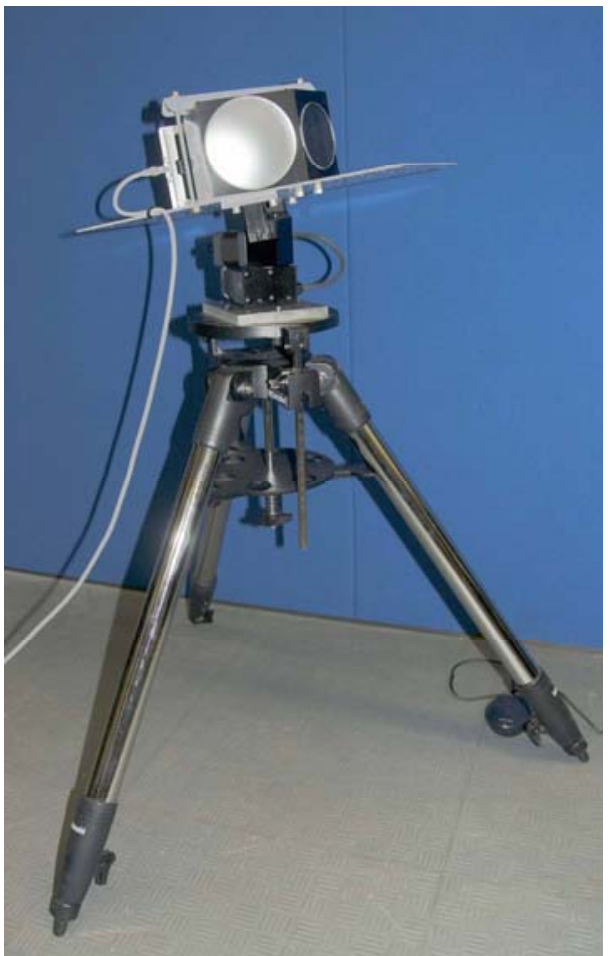

(b)

Figure 2. NASA LaRC IntelliStar Prototype.

\subsection{Active Optic Components for IntelliStar system}

The IntelliStar system shown in figure 2 does not include the active optic corrector plate and micro-mirrors which are currently under development. For the next generation IntelliStar system one of the active optic corrector plate technologies we are investigating is a liquid crystal phase modulator. The corrector plate illustrated in figure 3(b) will be approximately 2.7 inches in diameter and have more than 37 actuators since only low frequency Zernike aberrations need to be compensated. ${ }^{3-5}$

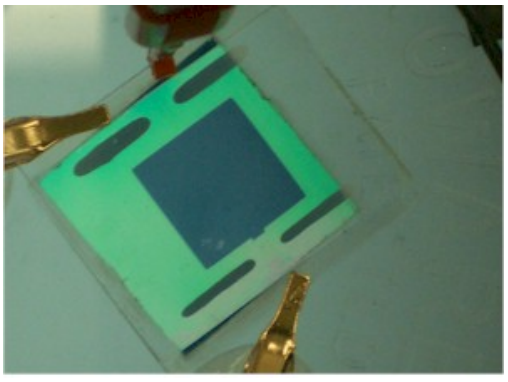

(a)

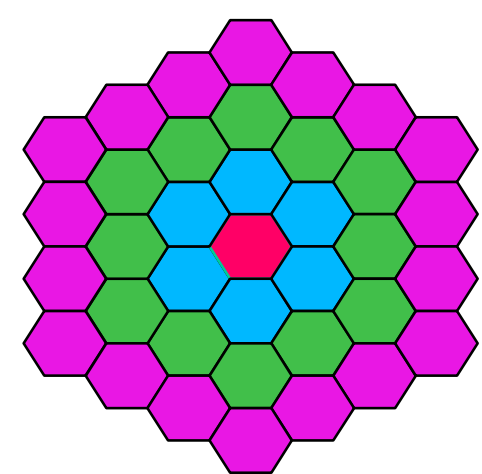

(b)

Figure 3. Liquid Crystal Phase modulator. (a) Single phase modulator cell fabricated in our NASA LaRC Intelligent Optical Systems clean-room and (b) Corrector plate architecture consisting of hexagonal single cell phase modulators. 


\subsection{IntelliStar Optical Head OTF and MTF}

The IntelliStar optical head has a 24 degree field of view. The point spread function of the IntelliStar optical head can be controlled by adding specific amounts of defocus blurring stars over a sub-array of pixels. Figure 3(a) shows the point spread function for an in-focus on-axis star for our IntelliStar system. Figure 5(a) is an image of Polaris star using the IntelliStar system with a small array active pixel sensor and figure 5(b) is a 24 degree field of view image of the Southern Cross using the Kodak 401 CCD sensor. Preliminary experimental results show that we should be able to achieve 0.2 arc second accuracy while spreading light over only four pixels. More accuracy can be achieved by spreading the light over more pixels. The irradiance falls to $1 / 4$ when the spot size is doubled as shown in figure 4. For our next generation star tracker we plan to incorporate corrector optics plate shown in figure 2 and deformable mirror technologies to provide tip/tilt and defocus so the star tracker can adapt to thermal effects causing mis-alignment and low order aberrations. In addition, the active optic technologies enable the star tracker to adapt to changing mission requirements and improve optical tracking performance. In the presence of bright stars within the field of view the star tracker can afford larger defocus and thus have higher tracking accuracy. If a higher bandwidth is needed for the spacecraft, the IntelliStar can reduce the defocus and yield higher bandwidth performance. Moreover, we are exploring how the IntelliStar System can be used as a multifunctional sensor for autonomous Rendezvous and Docking and distributed satellite systems. ${ }^{2-3}$

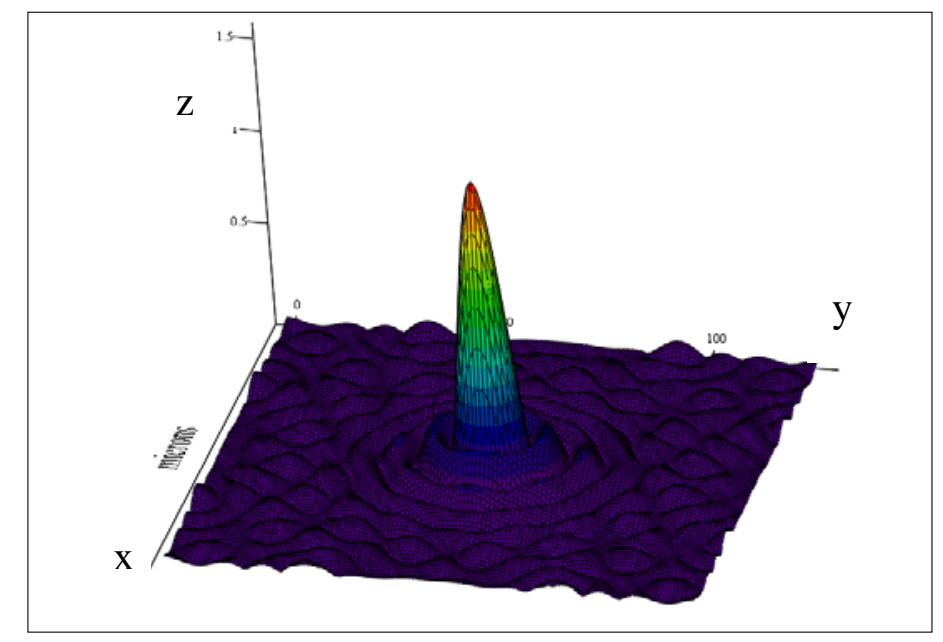

(a)



(b)

Figure 4. Point Spread Functions (PSF). (a) Best focus stars with Airy disc (PSF) size of 25 microns full width half max and (b) a diameter twice that of the in-focus Airy size (full width half max of 50 microns). The $\mathrm{x}$ and $\mathrm{y}$ axes are in microns and the $\mathrm{z}$ axis is the amplitude of the $\mathrm{PSF}^{4}$. 


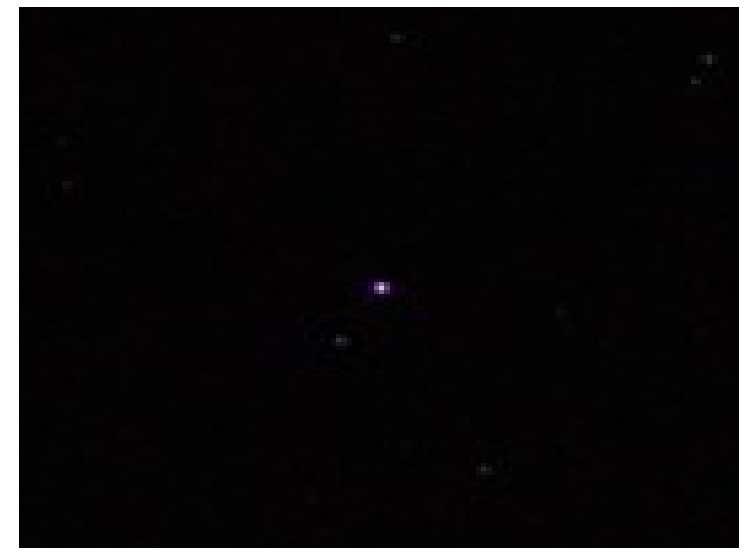

(a)

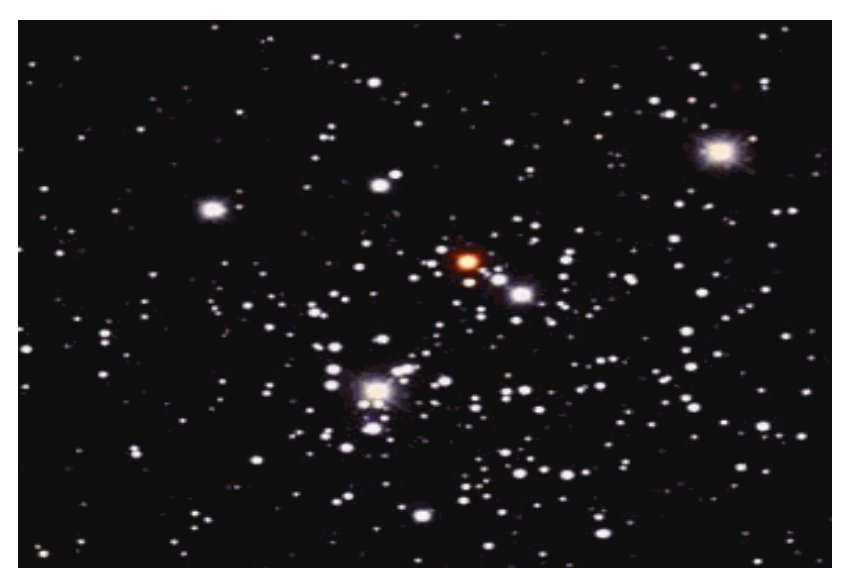

(b)

Figure 5. IntelliStar Image. (a) Image of Polaris source using IntelliStar on our small array of low light APS (b) wide 24 degree FOV obtained from the IntelliStar System using Kodak 401 CCD chip.

\subsection{Silicon Carbide}

The dramatic difference in weight between conventional and Silicon Carbide optical systems has led to Silicon Carbide material being applied to a number of optical applications associated with next generation remote sensing concepts. Silicon Carbide material has a number of bulk property advantages high specific stiffness and outstanding thermal stability which makes it particularly suited for the demanding space environment needed in star tracker systems. Silicon Carbide has a density approximately $80 \%$ the that of beryllium and is $50 \%$ the hardness of diamond. Consequently Silicon Carbide is lighter weight and strong material suitable for demanding space applications. Historically there are two problems associated with CVD Silicon Carbide materials(1) the CVD process is very expensive with furnacing runs costing on the order of \$100k each and (2) CVD SiC cannot be produced in very light weight geometries. A state of the art CVD Silicon Carbide mirror can be expected to have a density on the order of $30-50 \mathrm{Kg} / \mathrm{m}^{\wedge} 2$. Hence, any complex geometries that are required need to be machined in place. Such machining is costly and time consuming. We plan on using a castable form of $\mathrm{SiC}$ as a reflector substrate material. Cast Silicon Carbide parts are formed by pouring a slurry of Silicon Carbide powders and water into a reusable mold. The mold can be very complex, as required for the folded up catadiotic telescope. This enables the intricate folded telescope design to be formed directly without the need for costly and time consuming machining. Finally, this Silicon Carbide technique has excellent polishability since the mirrors can be formed with surface roughness less than lambda/20. Moreover, our analysis indicates that the optical head has a small recurring cost estimated to be under $\$ 15 \mathrm{~K} .^{1-4}$

\section{Active Pixels}

Current NASA and DoD efforts to develop an Active Pixel Sensor (APS) based star tracker have relied on commercially available optics and detectors developed to operate under bright daylight conditions. Efforts by NASA and DoD to retrofit or modify active commercial sensors have been disappointing because they do not operate well under demanding and low-light conditions. The IntelliStar APS sensor shown in figure 6 is a reconfigurable active pixel position sensor that enables low light, high dynamic range tracking and imaging to be accomplished in a single detector array that overcomes many of the problems associated with commercial detectors. The architecture of the large $1024 \times 1024$ array we are currently developing is described in section 2.1 and illustrated in figure 7. Our LaRC Active Pixel Sensor (APS) has several advantages over CCD and traditional Active Pixel Sensors. Our APS sensor offers the advantage of two orders of magnitude less power consumption and less susceptibility to radiation damage. Our LaRC APS can be directly accessed, simplifying the camera system design and enhancing its capabilities. Also like many APS sensors, our APS sensors are substantially cheaper to produce (in quantity) than traditional CCDs and allow for reduced component count. Figure 6 shows a schematic of an active pixel sensor we fabricated using the XFAB process along with an image of the resulting fabricated die of an $8 \times 8$ array of pixels. ${ }^{1-3}$ 


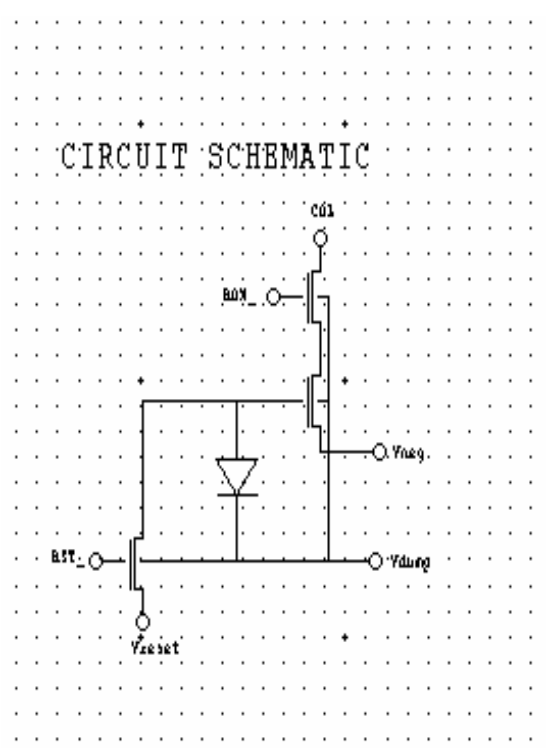

(a)

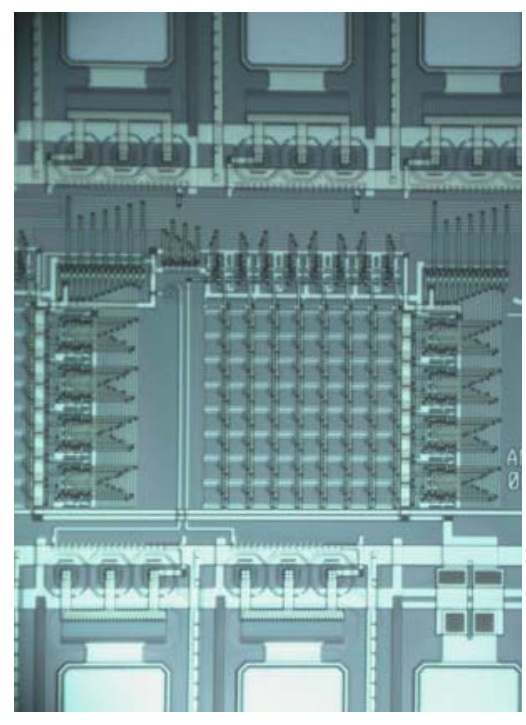

(b)

Figure 6. Active pixel sensor. (a) schematic of a single pixel (b) image of 8x8 CMOS APS array.

In order to test the active pixel sensor careful consideration of the radiometry of the stars, the IntelliStar optics, and earth testing conditions being different than those of space environment.

For the IntelliStar system we use the radiometric analysis to group stars into categories based on their magnitude. The magnitude of a star is an indication of its brightness. The greater the magnitude is, the fainter the star. The magnitude of a star is an arbitrary scale invented by the Greeks. According to them, the brightest stars in the sky had a magnitude 1 and the faintest had magnitude 6 . The same basic definition is now used, but with a more formal and mathematical meaning. Now we know that according to the psychophysical law of Fetchner ${ }^{6}$, the optical sensation in the eye is directly proportional to the logarithm of the luminous excitation. Based on this effect, John Herchel in 1830 defined that the first magnitude star is 100 times brighter than the sixth magnitude star. Thus, the brightness of a star of magnitude one (M1) is (100)^1/5=2.512 times larger. A useful relationship of two stars with magnitude $m$ and $n$ having brightness $B_{n}$ and $B_{m}$ respectively, we may express mathematically

$$
\frac{B_{n}}{B_{m}}=2.512^{m-n}
$$

Taking logarithms on each side yields

$$
\log B_{n}-\log B_{m}=(m-n) \log 2.512=0.4(m-n)
$$

Referring to equation (2) above, the magnitude difference between two stars is directly proportional to the difference in the logarithm of their brightness. The first magnitude star was then defined arbitrarily as the magnitude of one star close to the brightest stars. 
In addition the sky background is not absolutely dark. The sky brightness in the image of the IntelliStar telescope may be shown to be inversely proportional to the square of the focal ratio or F-number [4]. When testing the star tracking on earth it is important to consider the atmospheric turbulence. The angular diameter of all stars is the same for all telescopes in a given astronomical site, due to the atmospheric turbulence. Thus, the size of the image is directly proportional to the effective focal length. On the other hand, the amount of energy forming the image is directly proportional to the square of the aperture. The energy per unit area in the image is also inversely proportional to the square of the focal ratio F-Number. Our IntelliStar system has an F\#=1.8. The Polaris star shown in figure 5(a) has a magnitude of 2.7. We are in the process of doing more exhaustive testing of the IntelliStar in our laboratories at NASA under relevant space environment conditions including thermal and radiation effects. We will also be performing several field tests of our IntelliStar System.

\subsection{APS Architecture}

Our IntelliStar APS sensor incorporates several features tailored for use in the IntelliStar system. Figure 7 is diagram of the active pixel architecture. The figure shows a block of 8x8 pixels can be reset. This enables the integration time for regions containing bright and dim stars to be adjusted. The read out is a non-destructive readout allowing any pixel to read out and be monitored. The gain on the amplifier is switch able. The combination of these control signals enables a wide dynamic range of star light to be collected. The correlated double sampling and 10 bit A/D can be enabled or disabled thus allowing for more adaptability to mission requirements.



Figure 7. Architecture of the IntelliStar Active Pixel Sensor. 


\section{Tunable Filters}

Tunable filters play an important role in many optical systems. Our cleanroom has the capability of fabricating a wide range of tunable optical filters. Fabry-Perot filters generally fall into one of two basic categories. In the first, the distance between the parallel plates is controlled to tune the wavelength that will pass through the filter. Piezo control is most typical. Newer interferometers have employed silicon MEMS technologies to control the separation between the plates. This type of device typically has difficulty maintaining the necessary tight tolerance on the parallel orientation of the plates. Doing so is complex and expensive. Furthermore, piezo devices, in addition to requiring high voltages, have a relatively narrow operating temperature range. The voltage and thermal requirements of the piezo-electric devices impose difficult and labor-intensive manufacturing and assembly operations, adding cost to the devices and making them less reliable. The second category of tunable filters uses fixed parallel plates and tunes the refractive index of an electro-optic material that fills the cavity between the plates; when voltage is applied, the refractive index changes. Typical electrooptic devices use lithium niobate or liquid crystal and often require unreasonably high voltages to achieve a tiny fraction of the desired tuning range. The small value of the electro-optic coefficient is responsible for the high voltage and insufficient tunability and leads to large, bulky devices. In this section we present two optical tunable filters. One such filter, a Fabry Perot etalon filter is shown in figure 8(a) along with some experimental results characterizing its spectral performance in 8(b). We plan to us the tunable etalon array as part of a spectrally tunable detector array. Each MEMs Fabry-Perot Etalon can be independently tuned to only allow one spectral component to reach the photo-detector element. Hence the device can operate as a hyperspectral imager in which individual pixel elements can be independently tuned in real time. ${ }^{5-9}$

\subsection{Fabry Perot Spectrally Tunable Detector Array}

We are developing a new class of MEMS devices for space applications using components made with highly compliant polymeric materials as a principal design element. Typically in MEMS devices, electrostatic or piezo forces are used to bend or deflect one of the MEMS layers to produce the desired mechanical motion. Stiffer materials require higher voltages to achieve a given mechanical deflection, and traditional silicon-based materials are all extraordinarily stiff. The Compliant MEMS technology platform differs from conventional MEMS by adding a set of softer, more compliant, polymeric materials to the list of conventional rigid siliconbased materials used in MEMS. Adding these compliant materials to the list of materials that can be deposited, patterned, and etched greatly widens the design space. The compliant materials used in this new class of MEMS devices are as much as six orders of magnitude less stiff and can easily be tailored over a range of three orders of magnitude. Additionally, they can be deposited in a much broader range of layer thicknesses. This very wide range of flexibility expands the design space for MEMS devices far beyond what is possible with traditional silicon-based materials.

Fabry-Perot filters generally fall into one of two basic categories. In the first, the distance between the parallel plates is controlled to tune the wavelength that will pass through the filter. Piezo control is most typical. Newer interferometers have employed silicon MEMS technologies to control the separation between the plates. This type of device typically has difficulty maintaining the necessary tight tolerance on the parallel orientation of the plates. Doing so is complex and expensive. Furthermore, piezo devices, in addition to requiring high voltages, have a relatively narrow operating temperature range. The voltage and thermal requirements of the piezoelectric devices impose difficult and labor-intensive manufacturing and assembly operations, adding cost to the devices and making them less reliable. The second category of tunable filters uses fixed parallel plates and tunes the refractive index of an electro-optic material that fills the cavity between the plates; when voltage is applied, the refractive index changes. One of our Fabry-Perot components we are developing is the tunable optical filter shown in figure 8.

\subsection{Birefringent Tunable Optical Filters}

Liquid crystal tunable filters play an important role in many optical systems. Figure 9 shows a liquid crystal Solc tunable filter. Such tunable filter based on Solc and Lyot can simultaneously accommodate an extremely narrow bandwidth and a wide field of view. In our rendezvous and docking, lunar landing hazard avoidance, and star tracking wide field of view tunable filters offer advantages for weak signals when extended sources are detected against a nosy background. Optical communications require a filter with not only an extremely narrow bandwidth to reject unwanted background light and hence increase the signal to noise ratio, but also a large angular aperture to receive as much signal as possible. 


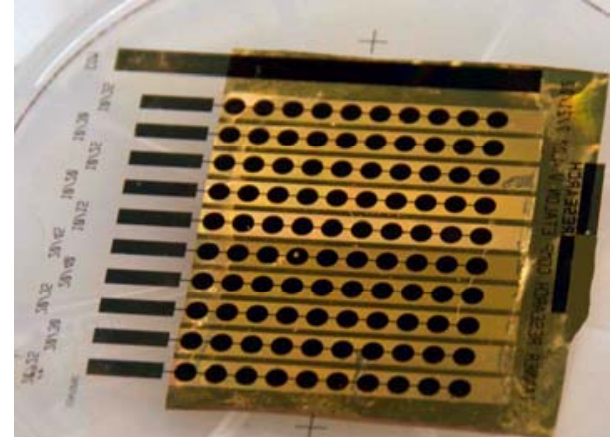

(a)

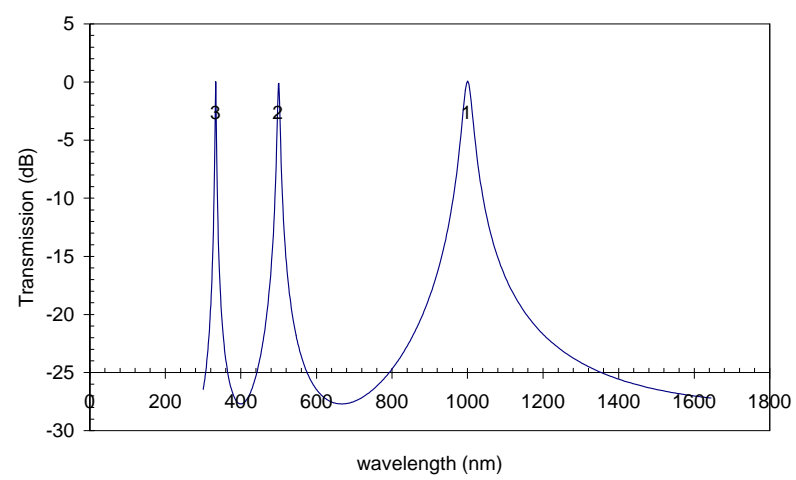

(b)

Figure 8. Tunable optical filters (a) Fabry Perot etalon and (b) spectrum

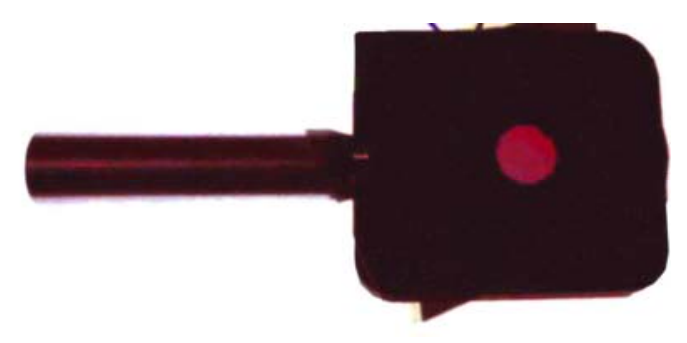

(a)

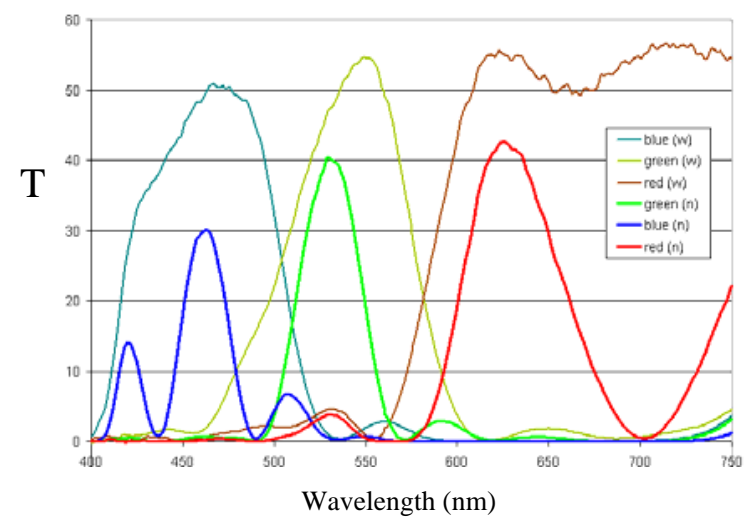

(b)

Figure 9. Liquid crystal tunable filter (a) and the spectral transmission (T). 


\section{Applications of the Technologies}

NASA has a need for a wide range of active optic devices for Space Exploration and Science Missions. Our sensors and actuators that support autonomous operations, cross cut several of NASA's key technical challenge areas including the Exploration Systems Architecture and Solar System Exploration Roadmaps. As discussed below a summary of the active optic devices we are developing for the Autonomous Landing and Hazard Avoidance Technologies (ALHAT); Decadal Survey Missions; and Autonomous Rendezvous and Docking (AR\&D) programs. The active optic technologies can also address many other programs of future Space Exploration including AR\&D for robotic assembly and servicing of the Crew Exploration Vehicle (CEV), and rendezvous and docking of staged ascent and landing vehicles in lunar and mars orbit. Our APS sensor could also enable robotic cargo transfer missions to the ISS, and robotic servicing and de-orbit of Hubble Space Telescope (HST). ALHAT and Decadal survey missions both need high precision 3D ATP for their next generation flash ladar ranger/imaging sensor suite and high precision image stabilization, platform jitter reduction. This effort also supports the Aeronautics Research Mission Directorate (ARMD) subsonic fixed wing Program by providing a testbed for novel polymers under development for morphing and large space optics.

\subsection{Guidance Navigation and Control}

To date the technical readiness level of navigation sensors is low and has been identified as one of the key space exploration risk areas associated with the Exploration Systems Architecture. Our IntelliStar system is key to GNC technology we are developing for Space Exploration missions. Star trackers are used for both military and nonmilitary applications on space probes, space-based interceptors, and satellites. In these applications precise attitude measurements are required. Instruments like our IntelliStar system provide a precision reference measurement for acquisition, tracking and pointing. On space missions, star trackers are currently the only sensor presently available that can provide arc-second attitude accuracy.

\subsection{Autonomous Rendezvous and Docking.}

During manned and unmanned missions, photo-sensors have provided critical decision-making data crucial to Mission success. Sensors are especially crucial during the spacecraft maneuvers for Rendezvous and Docking (R\&D). In the course of developing these sensors NASA realized that a system was needed for Automated Rendezvous and Docking (AR\&D) to act as a potential backup system for manned missions or as a primary capability on unmanned missions. With the recent advent of NASA's Exploration Initiative, slated to return to Lunar Exploration and eventual human exploration of Mars, NASA's work with AR\&D has been elevated in importance. This is due to the fact that future Lunar and Mars exploration will rely heavily on the assembly of large elements, in low earth orbit and beyond. At present, R\&D technology requires extensive human intervention for flight operations and ground control; however, data latency for operations on Mars precludes ground involvement for proximity operations and becomes very problematic for lunar operations. Therefore, an AR\&D system, with less reliance on human participation (both ground and crew) is considered to be necessary to accomplish the goals of the Exploration Initiative.

\subsection{Autonomous Landing and Hazard Avoidance Technology (ALHAT)}

Future lunar missions will need to land near specific resources that are located in potentially hazardous terrain. This capability will be possible when landers have with the ability to automatically recognize the location of the desired landing site while detecting landing hazards during the final descent to the surface. Two critical technologies that must be developed to enable this capability are an active sensor for measuring the topography of the landing site and terrain analysis algorithms. To prove that these technologies are ready for flight, they must be tested using both field tests and high fidelity Monte Carlo simulations of lunar landings. In addition, the sensing and terrain analysis algorithms must be tested in the field to prove their performance in a relevant environment and to validate software models using simulations. The final phase of testing takes place in a Hardware-in-the-Loop testbed where the landing dynamics, lunar environment, sensors, and software are all present for full up real-time system testing. This five year task is part of the Autonomous Landing and Hazard Avoidance Technology (ALHAT) program led by Johnson Space Center (JSC) with support from the Jet Propulsion Laboratory (JPL), Langley Research Center (LaRC) and Charles Stark Draper Labs (CSDL). We are 
investigating use of our APS sensor technologies using active optic devices for a high bandwidth novel flash LADAR system for ALHAT and the DoD.

\section{Summary}

NASA Langley Research Center is involved in the development of photonic devices and systems for space exploration missions. Photonic technologies of particular interest are those that can be utilized in our Space Exploration Architecture roadmap. Applications include in-space communication, remote sensing, guidance navigation and control, lunar descent and landing, and rendezvous and docking. NASA Langley has recently established a class-100 clean-room which serves as a Photonics Fabrication Facility for development of prototype optoelectronic devices for aerospace application. In this paper we discuss our design, fabrication, and testing of novel active pixels, deformable mirrors, and liquid crystal spatial light modulators. The active pixel sensor we presented was designed as a multifunctional sensor for the IntelliStar system and for autonomous rendezvous and docking. We fabricated two types of tunable filter devices. One of the tunable filters presented was liquid crystal based and the second tunable filter was a novel polymer based Fabry Perot Etalon. The design considerations for the tunable filters were presented along with experimental results characterizing their performance. Both tunable filters offer advantages in space exploration. Fabry Perot Etalons are often used when a very narrow tuning band is required and the Liquid crystal tunable filters are often used with a wide field of view. Liquid crystal tunable filters offer the advantage that they can also be designed to have more complex spectral transmissions that can be controlled in real time. The IntelliStar system presented is a complex electrooptical system utilizing active pixels, deformable mirrors and liquid crystal corrector plates. The use of active optics allows the IntelliStar system to be reconfigured in real time.

Successful implementation of these intelligent optical devices and systems in space requires careful consideration of temperature and space radiation effects in inorganic and electronic materials. Applications including high bandwidth inertial reference units, lightweight, high precision star trackers for guidance, navigation, and control, deformable mirrors, wavefront sensing, and beam steering technologies were discussed. In addition, experimental results are presented which characterize their performance in space exploration systems.

\section{References}

1. N.Clark and P.Furth, "Silicon eye using MEMs Micromirrors”, Optics and Photonics News, vol. 11, No. 11, pp. 34-38, November 2000 .

2N. Clark, P.Furth, and S. Horan, “Intelligent Star Tracker," $14^{\text {th }}$ Annual/USU Con Conference on Small Satellites, Logan, Utah, August 2000.

3. N.Clark and P. Furth, The and P. Furth, "Design and performance evaluation of a silicon eye using micromirrors,” The 43rd Midwest Symposium on Circuits and Systems, Lansing, MI, August 2000.

4. J.D. Gaskill, Linear Systems, Fourier Transforms and Optics, Wiley and Sons, New York, NY (1978).

5. I. Solc, “Birefringent Chain Filters,” Journal of the Optical Society of America, 55, 621 (1965).

6. Herrmann, D. B. 1984, “The History of Astronomy from Herschel to Hertzsprung,” translated

By Kevin Krisciunas (Cambridge: Cambridge Univ. Press)

7. J. W. Evans, “The birefringent filter” Opt. Soc. Am. 3, 229-242 (1949).

8 S. E. Harris, E. O. Amman, and I. C. Chang, “Optical network synthesis using birefringent crystals. I. Synthesis of lossless networks of equal-length crystals,” J. Opt. Soc. Am. 10, 1267-1279 (1964).

9. EO. Ammann, “Optical network synthesis using birefringent crystals,” J. Opt. Soc. Am. 56, 952-955 (1966).

10. P.M. Furth and N.Clark, “Analog VLSI subaperture centroid circuits,” $7^{\text {th }}$ NASA Symp VLSI Design, Albuquerque, NM October 1998. 